\title{
Les déclarations de Bâle : mises à jour et pertinentes pour tous
}

\author{
par Rebekah J. Moles, Lee Vermeulen, Jonathan Penm et Marianne Ivey
}

$\mathrm{E}$ n 2008, la Fédération internationale pharmaceutique (FIP) organisait la Conférence mondiale sur l'avenir de la pharmacie hospitalière à Bâle en Suisse. Le résultat de cette rencontre historique a été la publication de 75 déclarations consensuelles, appelées les déclarations de Bâle, qui représentent une vision commune de la pratique de la pharmacie hospitalière ${ }^{1}$. En 2014, des leaders et des praticiens de pharmacie hospitalière ont entrepris une vaste révision de ces déclarations afin de s'assurer qu'elles demeurent actuelles et applicables à la pratique. La révision comprenait trois phases : un sondage mondial en ligne, une analyse réalisée à l'aide d'un forum en ligne et un atelier en mode " conversation de café " (appelé aussi " café du monde " ou World Café).

Lors de la première phase, à la fin 2013 et au début 2014, un sondage a été présenté aux parties prenantes en pharmacie hospitalière. Les participants étaient invités à commenter chacune des déclarations soit : en affirmant leur accord avec la formulation de la déclaration, en proposant qu'elle soit éliminée ou en suggérant qu'on la reformule. Les répondants qui sélectionnaient la troisième option avaient la possibilité de suggérer un choix de mots. Les répondants pouvaient aussi soumettre des déclarations entièrement nouvelles. Au total, 334 réponses au sondage provenant de 62 pays ont été obtenues. Plus de $10 \%$ des répondants ont jugé que 14 déclarations devaient être reformulées ou éliminées. Ces dernières ont fait l'objet de discussions rigoureuses lorsque la direction de la Section de pharmacie hospitalière s'est réunie en personne à la fin mars 2014. Après des révisions initiales par la direction, les déclarations ont été soumises à une analyse plus approfondie au moyen d'un forum mondial en ligne. Au cours de cette phase, des personnes de 28 pays, représentant l'ensemble des six régions de l'Organisation mondiale de la santé, ont fait des recommandations. Cet exercice a abouti à l'apparition de nouveaux concepts dignes d'intérêt, notamment le rôle de l'évaluation des technologies, la responsabilité des pharmacies hospitalières en ce qui touche à l'intégrité des produits stériles préparés en sous-traitance et la formation de pharmaciens hospitaliers spécialistes. Lors de la dernière phase, 80 participants de 20 pays ont pris part à un atelier en mode " conversation de café " au congrès de la FIP en 2014 à Bangkok. À la fin de l'atelier, les participants se sont prononcés sur les déclarations, ce qui a abouti à l'adoption des 65 déclarations de Bâle révisées finales, qui s'inscrivent sous les mêmes six thèmes que les déclarations d'origine : approvisionnement, influences sur la prescription, préparation et délivrance des médicaments, administration des médicaments, surveillance des pratiques relatives aux médicaments ainsi que ressources humaines et formation. Un ensemble de déclarations primordiales est aussi disponible. En septembre 2015, les déclarations de Bâle révisées (disponibles au www.fip.org/basel-statements) ont été rendues publiques pour le monde entier.

Mais alors, qu'est-ce qui a changé? Pour ceux qui avaient déjà commencé à adopter les déclarations d'origine, la bonne nouvelle est qu'ils peuvent continuer : même si les déclarations sont moins nombreuses, l'essence de ce qu'elles affirment demeure intacte. En fait, la réduction du nombre de déclarations s'explique surtout par l'identification et l'élimination de répétitions. Cela dit, de nouveaux concepts ont aussi été ajoutés, particulièrement en ce qui touche à la gestion des effets des médicaments sur l'environnement, aux avancées des technologies de l'information et à l'utilisation des dossiers de santé informatisés. De plus, des termes utilisés par la FIP (" utilisation responsable des médicaments " par exemple) sont incorporés dans les nouvelles déclarations. Finalement, les changements de formulation visent à simplifier les déclarations, à les rendre ainsi plus claires et plus faciles à appliquer, peu importe le pays.

Grâce à ces assurances sur le bien-fondé des changements, vous pourriez être amené à croire que disposer de cet ensemble de déclarations est très bien, mais vous pourriez malgré tout vous demander si elles sont vraiment pertinentes. Vous pourriez aussi vous demander comment elles peuvent être utilisées. La direction 
de la Section de pharmacie hospitalière de la FIP croit que ces déclarations offrent une feuille de route pour la pratique de la pharmacie hospitalière, car elles ont une portée véritablement mondiale et qu'elles reflètent des normes idéales. Depuis leur création en 2008, les déclarations de Bâle ont été utilisées par les organismes de pharmacie hospitalière dans différents pays et par des hôpitaux dans le but de transformer ou de surveiller la pratique. Certains établissements pourraient même se servir des déclarations pour « tirer parti » des infrastructures politiques en vue de soutenir l'adoption de services de pharmacie additionnels.

Le présent numéro du Journal canadien de la pharmacie hospitalière (JCPH) contient un examen de la portée que revêtent les déclarations de Bâle en recherche ${ }^{2}$. Pour réaliser leur examen, les auteurs ont recensé 14 articles publiés : quatre traitant de l'adaptation des déclarations au contexte européen, six concernant l'utilisation des déclarations pour la surveillance de la pratique actuelle et quatre abordant les éléments qui font obstacle ou qui facilitent la mise en ouvre de certaines déclarations. Bien qu'une grande partie des recherches réalisées à ce jour le furent dans la région du Pacifique occidental et en Europe, l'examen de la portée présente aussi une étude longitudinale menée dans un hôpital en Uganda et décrite dans un article précédent du $J C P H^{3}$. Les auteurs de ce rapport antérieur ont souligné des améliorations apportées à la mise en œuvre des déclarations au fil du temps. Il semble que ce qui a encouragé le changement était une connaissance croissante des déclarations et une volonté du personnel de la pharmacie de s'appliquer à améliorer les services en fonction des déclarations ${ }^{3}$. Selon ces observations, plus ces déclarations sont diffusées, lues, considérées et surveillées, plus il y a de chances qu'on observe des changements rapides dans la prestation des services de pharmacie hospitalière partout dans le monde. Il revient donc à tous les pharmaciens d'hôpitaux de lire les déclarations et de les faire connaître à leurs collègues.

Les praticiens hospitaliers de pays développés semblables aux nôtres (l'Australie et les États-Unis), qui possèdent des façons de faire similaires à celles au Canada, pourraient être tentés de ne jeter qu'un oil distrait aux déclarations consensuelles de Bâle. Ils pourraient croire que ces dernières ne sont pas nécessaires, que la pratique en place convient bien et qu'ils agissent au meilleur de leur capacité avec les ressources disponibles. Néanmoins, nous vous incitons à bien lire ces déclarations, à en trouver que vous ne suivez peut-être pas aussi bien que vous le pourriez et à réfléchir sur les changements que vous pourriez apporter à votre milieu de travail. À l'inverse, si vous faites déjà très bien quelque chose tout en adhérant à l'une des déclarations, pourquoi ne pas raconter aux établissements et aux praticiens d'ici et d'ailleurs comment vous avez aidé votre service de pharmacie à atteindre un tel niveau de performance? Afin de prêcher davantage la bonne parole, nous recommandons aussi aux organismes de pharmacie de planifier des présentations afin de parler de leurs succès liés à l'utilisation des différentes déclarations de Bâle. En tant que membres de la direction de la Section de pharmacie hospitalière de la FIP, nous sommes convaincus que ces déclarations sont pertinentes pour tous et qu'elles permettront de maximiser la valeur qu'apportent les pharmaciens hospitaliers au bien-être de leurs patients. Nous vous encourageons donc à étudier les déclarations et à prendre des mesures pour qu'elles se concrétisent dans votre milieu de travail!

[Traduction par l'éditeur]

\section{Références}

1. The Basel Statements on the future of hospital pharmacy. Am J Health Syst Pharm. 2009;66(3):S61-6.

2. Penm J, Chaar B, Moles RJ. Use of the International Pharmaceutical Federation's Basel statements to assess and advance hospital pharmacy practice: a scoping review. Can J Hosp Pharm. 2016;69(2):131-7.

3. Poh J, Vaillancourt R, Lamarre D, Oyela J. Use of the 2008 Basel consensus statements to assess, realign, and monitor pharmacy practice at a tertiary care hospital in northern Uganda: illustrative case study. Can J Hosp Pharm. 2013;66(5):318-27.

Rebekah J. Moles, B. Pharm., Dip. Hosp. Pharm., Ph. D., Grad. Cert. Ed. Stud. (Higher Ed.), travaille à la Faculté de pharmacie de I'Université de Sydney, Sydney, Nouvelle-Galles du Sud, Australie. Elle est également rédactrice adjointe du $J C P H$.

Lee Vermeulen, R. Ph., travail au Center for Clinical Knowledge Management, UW Health de I'Université du Wisconsin-Madison, Madison, Wisconsin.

Jonathan Penm, Ph. D., travaille à la Faculté de pharmacie James L Winkle de I'Université de Cincinnati, Cincinnati, Ohio.

Marianne Ivey, Pharm. D., travaille à la Faculté de la pratique de la pharmacie et des sciences administratives de I'Université de Cincinnati, Cincinnati, Ohio.

Intérêts concurrents : Tous les auteurs font partie de la direction de la Section de pharmacie hospitalière de la Fédération internationale pharmaceutique. Les auteurs n'ont déclaré aucun autre intérêt concurrent.

Address correspondence to:

De Rebekah J Moles

Faculty of Pharmacy

The University of Sydney

Pharmacy and Bank Building A15

Sydney, NSW 2006 Australia

Courriel : rebekah.moles@sydney.edu.au 